\title{
Design of HPCF with nearly zero flattened Chromatic Dispersion
}

\author{
Ravindra Kumar Sharma, Sunil Sharma, Shambhu Dayal Sharma
}

\begin{abstract}
Here we have consider an air-silica honeycomb lattice and demonstrate a new approach to the formation of a core defect. The honeycomb lattice has recently been suggested for the formation of a photonic band-gap guiding silica-core. Here we discuss how a core defect can be formed by manipulating the cladding region rather than the core region itself. There are so many difficulties occur during the design process. Because when parameters are being changed the designing varies accordingly. For this purpose we have use HPCF in scalar effective index method (SEIM) and consider chromatic dispersion of HPCF for having air hole diameter 1.0 $\mu \mathrm{m}$, and pitch $2.0 \mu \mathrm{m}$. The maximum pitch can be consider as $\mathbf{5 . 0}$ $\mu \mathrm{m}$ and air hole diameter is $9.0 \mu \mathrm{m}$. Transparent boundary condition (TBC) is proposed here for the calculation of Refractive index of HPCF.
\end{abstract}

Index Terms - Honeycomb PCF, Ultra flattened Dispersion, TBC, SEIM. Refractive index.

\section{INTRODUCTION}

In the recent scenario the photonic crystal fiber have attracted much attention because it exhibits many unique optical properties, such as single-mode operation over a wide wavelength range, large waveguide chromatic dispersion, or a high degree of freedom in design. Now a days, research is carried on photonic crystal fibers (PCFs) which are also called holey fibers $[1,2]$. The PCFs have a central region called core surrounded by periodic air holes which work as a cladding. The PCF technology is used to create a fiber with high nonlinear coefficient and zero chromatic dispersion.

Most photonic crystal fibers (PCFs) have been fabricated in silica glass.

The value of refractive index of silica glass can be calculated by sellemier formula.

$$
\boldsymbol{n}^{2}-1=\sum_{i}\left(\frac{\boldsymbol{A}_{i} \lambda^{2}}{\lambda^{2}-\lambda_{1}^{2}}\right)
$$

And the total dispersion, $\mathrm{D}=\mathrm{D}_{\mathrm{M}}+\mathrm{D}_{\mathrm{W}}$. and the waveguide dispersion is defined as

Manuscript received November 09, 2014

Ravindra Kumar Sharma is Assistant professor in Rajadhani Institute of Technology \& Management. Jiapur and completed his M.Tech. in Digital Communication System from Rajasthan Technical university, Kota. 9414437778

Sunil Sharma is M.Tech. Scholar from Rajasthan Technical University, Kota. He is Pursuing is M.Tech. in Digital Communication. 9785740162.

Shambhu Dayal Sharma, Pursuing in M.Tech., MNIT, Jaipur.

$$
D_{w}=-\left(\frac{\lambda}{c}\right) \frac{d^{2}}{d \lambda^{2}} n_{e f f}
$$

Where $\lambda$ is the operating wavelength and $\mathrm{c}$ is the velocity of light.

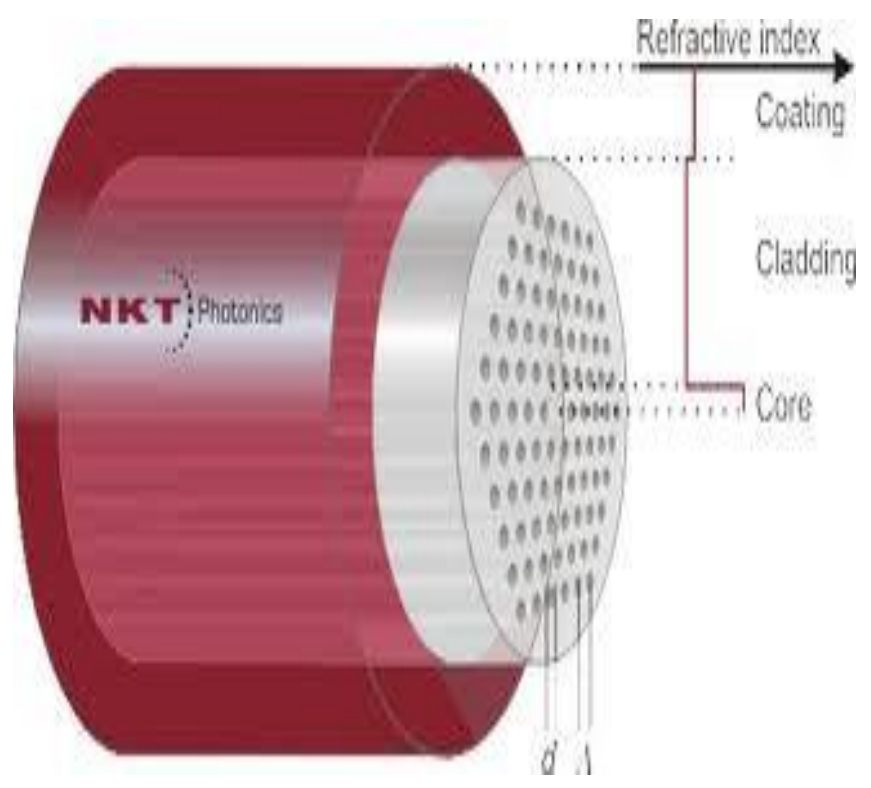

Figure: 1. Photonic Crystal Fiber [15]

In this paper, the optical properties of solid core HPCF with different up/down doping levels are considered. The doping level of the core adds new freedom of solid core HPCF design.[3-5]. In optical communication, dispersion plays an important role as it determines the information carrying capacity of the fiber. Therefore it becomes important to study the dispersion properties of PCF.[6] To improve the accuracy different values for the effective core radius are used. The results show that the solid core HPCF has great potentials and dispersion compensation for air hole diameter $\mathrm{d}=1.0 \mu \mathrm{m}$, Pitch $=2.0 \mu \mathrm{m}$ and layer $=7$.

The optical properties of PCF have been used like Effective Index method (EIM), Scalar effective index method (SEIM).[3] The SEIM method is used for weak guiding approximation and accurate approximations. In the SEIM method the value selection for effective core radius of the PCF is important for the accuracy.[6,7] We calculate the effective refractive index of the fundamental mode $n_{\text {eff }}$ of the PCF in the commonly used SEIM model the relative hole diameter $\mathrm{d} / \Lambda$ varying from 0.2 to 0.8 . 
The accuracy of this method is worth investigating as the weakly guiding approximation in the SEIM model is made invalid by the large index contrast between silica and air. The definition of the radius of the unit cell in calculating the effective cladding index and the fiber core of the corresponding approximate step-index fiber remain ambiguous and different values are used. [8-11]The novel fiber has a central low-index core region and a cladding consisting of a silica background material with air holes situated within a honeycomb lattice structure. The results for a honeycomb-based silica-air photonic crystal fiber are in very good agreement with the results from the other methods.[12]

\section{SCALAR EFFECTIVE INDEX METHOD}

In SEIM the effective cladding index of a hexagonal unit cell which consists a Fiber rod is calculated with respect to rod diameter and pitch $(\Lambda)$, then the effective index of PCF is obtained by using the effective cladding index.[16]

One of the best suited analytical methods is scalar effective index method which is valid for the LP01 fiber mode based on weak-guidance approximation. Fundamental space-filling mode (FSM) of a PCF is considered to be the mode with the largest modal index of the infinite two-dimensional photonic crystal structure that surrounds the PCFs core.

The core refractive index is supposed to be the same as the refractive index of core material which is given by the Sellmeier formula. But the cladding refractive index is determined based on total reflection.[19].

The Scalar wave equation is :

$$
\left[\Delta_{\mathrm{t}}^{2}+\left(\mathrm{k}^{2} \mathrm{n}^{2}-\beta^{2}\right)\right] \Psi
$$

Used to obtain the effective cladding index. Here $\boldsymbol{\Delta}_{\mathbf{t}}$ is the transverse laplacian operator in cylindrical coordinates, $\mathrm{k}=(\omega / \mathrm{c})$ is the wave number in Vacuum, $\mathrm{n}$ is the material index, and $\beta$ is the propagation constant and $\psi$ can be either the electric or magnetic field.

We can get the following equation in the inner and outer areas of the air hole under the assumption of weak guidance.

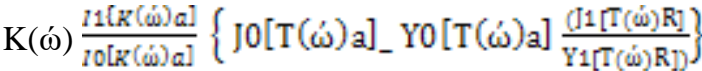

Equal to

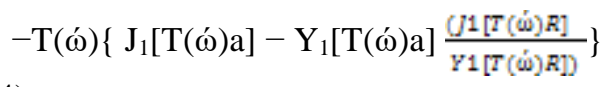

Where I, J, and $\mathrm{Y}$ are Bessel functions. The optimum radius for $\mathrm{SEIM}$ is $\mathrm{R}=\Lambda / 2$.[16]

In terms of $\beta(\dot{\omega})$ we can find out the effective refractive index for Space filling mode. The hexagonal unit cell is approximated by a circular one of radius $\mathrm{R}$.

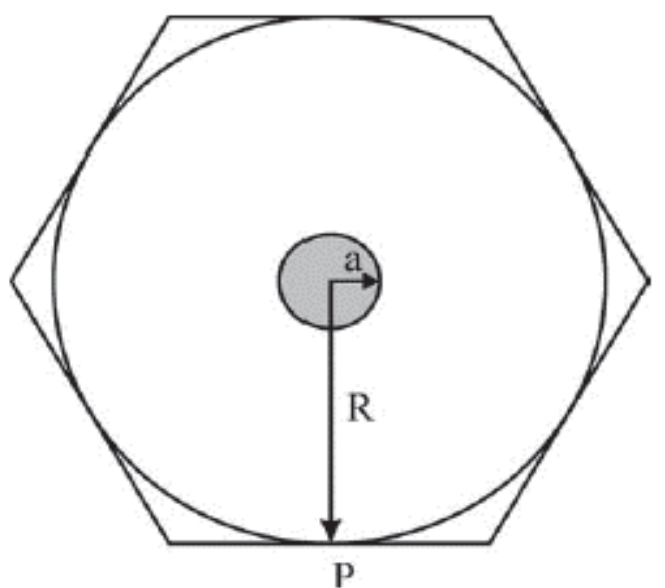

Figure: 2. circular unit cell of hexagonal [16]

\section{DESIGN PRINCIPLE}

In this method consider that refractive index of silica is $\mathrm{n}_{\text {core }}$ $=1.45$ and the effective core radius is $\mathrm{a}_{\mathrm{eff}}=\Lambda /(3)^{0.5}$.

It is consider that the triangular PCFs can be well parameterized in terms of the $\mathrm{V}$ parameter and from $\mathrm{V}$ parameter we have

$\mathrm{V}=\frac{2 \pi}{\lambda} \mathrm{a}_{\mathrm{eff}}\left(\mathrm{n}_{\text {core }}^{2}-\mathrm{n}_{\text {fsm }}^{2}\right)^{0.5}=\left(\mathrm{U}^{2}+\mathrm{W}^{2}\right)^{0.5}$

Where $\mathrm{U}=\frac{2 \pi}{\mathrm{\lambda}} \mathrm{a}_{\mathrm{eff}}\left(\mathrm{n}^{2} \text { core }-\mathrm{n}^{2} \text { fsm }\right)^{0.5}$ and

$$
\mathrm{W}=\frac{2 \pi}{\lambda} \mathrm{a}_{\mathrm{eff}}\left(\mathrm{n}_{\mathrm{eff}}^{2}-\mathrm{n}_{\mathrm{fsm}}^{2}\right)^{0.5}
$$

The values of

$\mathrm{k}^{2}(\dot{\omega})=\boldsymbol{\beta}^{2}(\dot{\omega})-\mathbf{n}^{2}$ air $\dot{\omega}^{2} / \mathrm{c}^{2}$

$\gamma^{2}(\dot{\omega})=\mathrm{n}_{\text {silica }}(\dot{\omega}) \dot{\omega}^{2} / \mathrm{c}^{2}-\boldsymbol{\beta}^{2}(\dot{\omega})$.

With c having the velocity of light in vacuum.

When we have solve the fundamental equation of SEIM for the propagation constant $\boldsymbol{\beta}(\dot{\omega})$ of the $\mathrm{LP}_{01}$ mode of approximate step index fiber

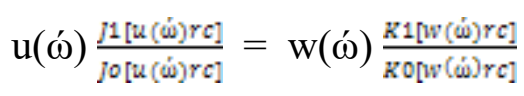

Where $\mathrm{n}_{\mathrm{c}}(\dot{\omega})$ is the refractive index of the core and $r_{c}$ is the core radius.

We have calculated fundamental space filling mode and the index guiding mode for different air hole sizes. Calculations have been done by using PCF parameters, hole to hole distance $\Lambda=2.0 \mu \mathrm{m}$, core radius $r_{c}=0.64 \mathrm{~L}$, air hole diameter $\mathrm{d}=1.0 \mu \mathrm{m}$. 
It is clear from the figure that as the air filling fraction increases the credibility of the SEIM reduces as the difference between the refractive index of the silica and the refractive index of the fundamental space filling mode increases, which is the foremost requirement of the weakly guided approximation.

\section{DISPERSION}

It is phenomenons in which the phase velocity of a wave depends on its frequency From the calculation of dispersion for various combination we have find that the proposed Honeycomb PCF structure is design as shown in figure 3 . The dispersion coefficient $\mathrm{D}(\Lambda)$, which include both waveguide and material dispersion, is proportional to the second derivative of effective index of guided mode with respect to ' $\Lambda$ '.

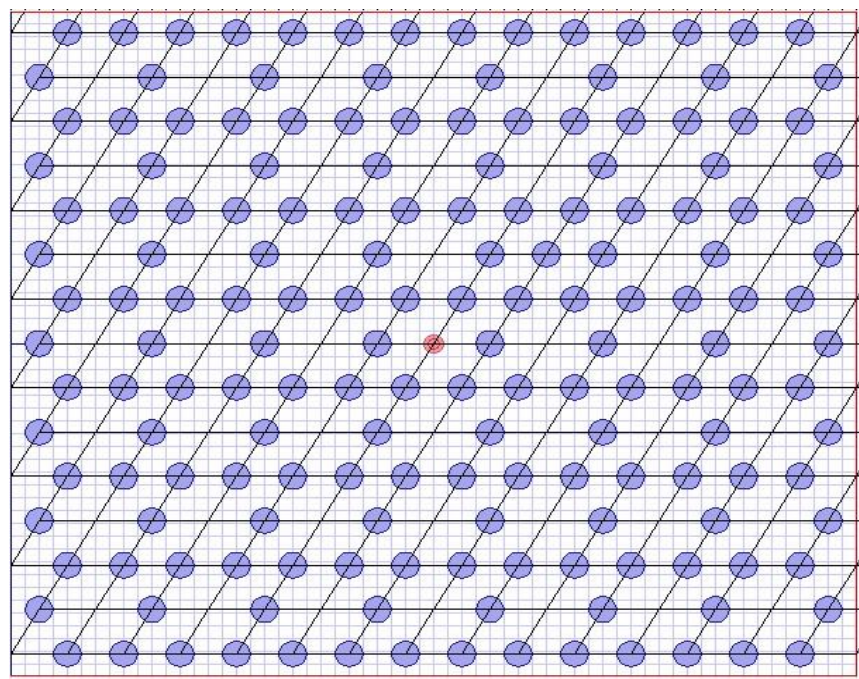

Figure: 3. Proposed Structure of Honeycomb PCF

This structure is designed for seven layer structure and having $\mathrm{d}=1.0 \mu \mathrm{m}$. The 2- $\mathrm{D}$ mode field pattern of Proposed Honeycomb PCF is given as:

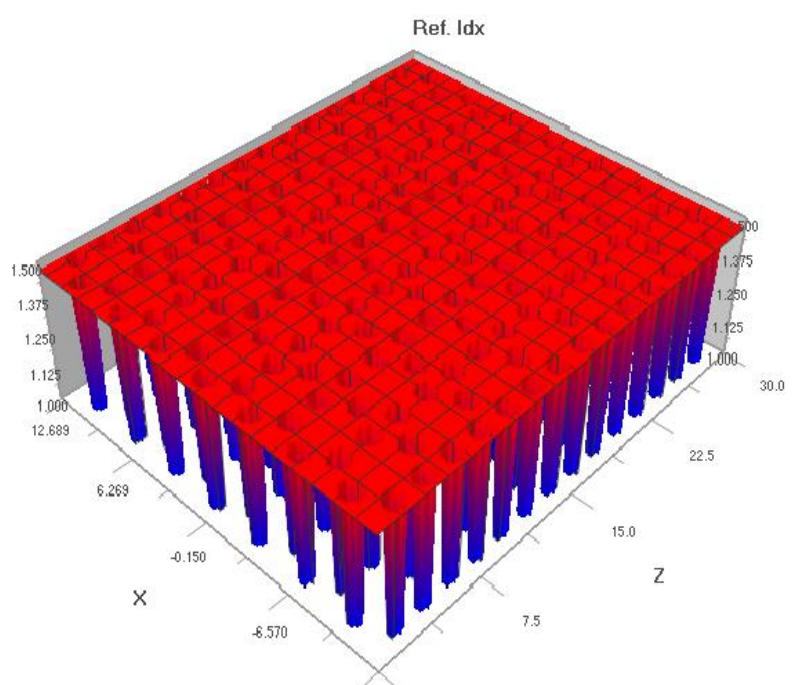

Figure: 4. 3-D layout design of HPCF

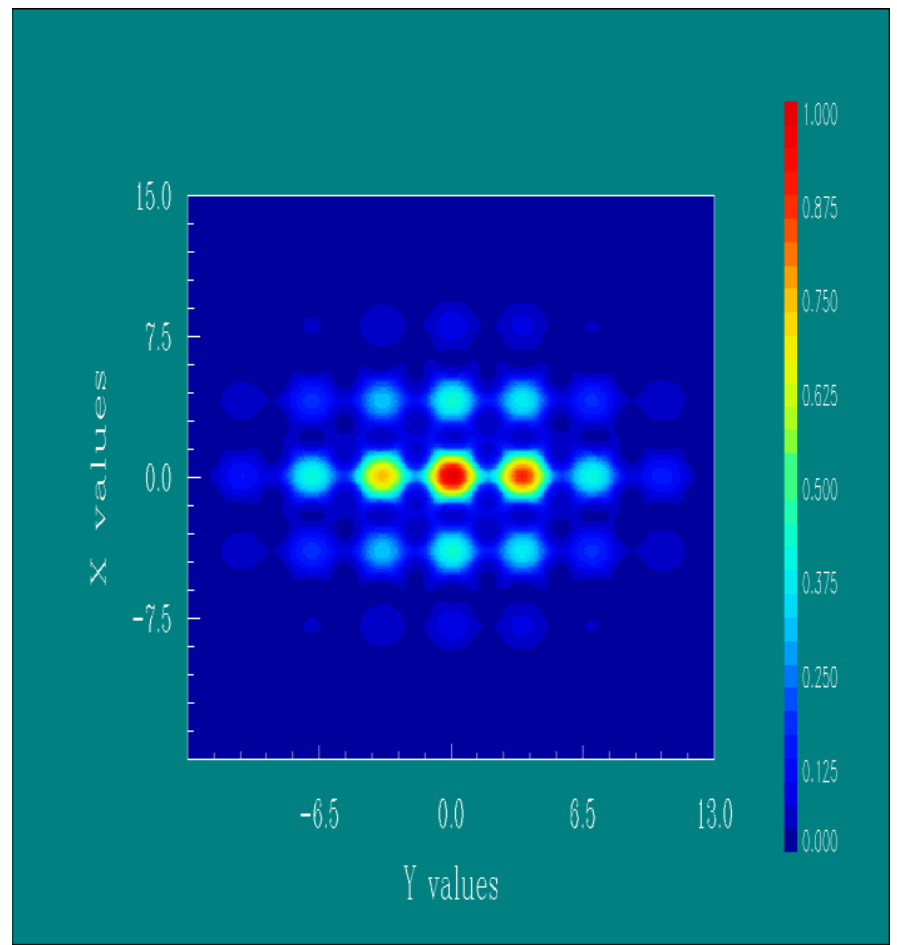

Figure: 5. D field pattern of HPCF

The Effective refractive index for silica glass HPCF is calculated and presented here for constant Pitch but having varying air hole diameter.

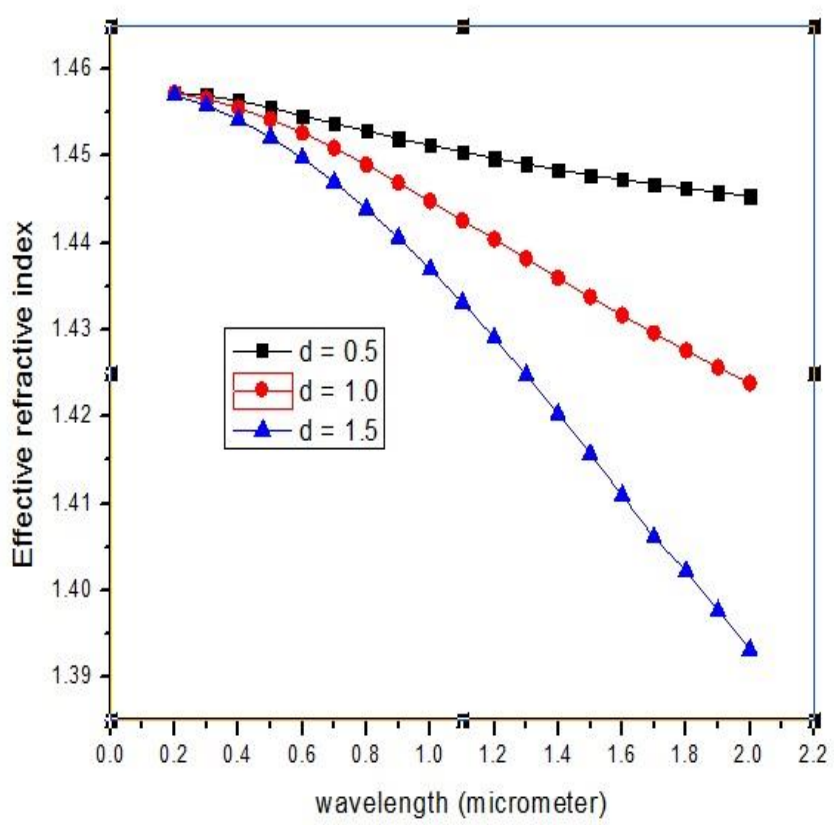

Figure: 6. Comparison of Effective refractive index for constant Pitch $($ Pitch $=2.0 \mu \mathrm{m})$

After this the calculation is carried out for constant air hole diameter but this time Pitch is varying. 


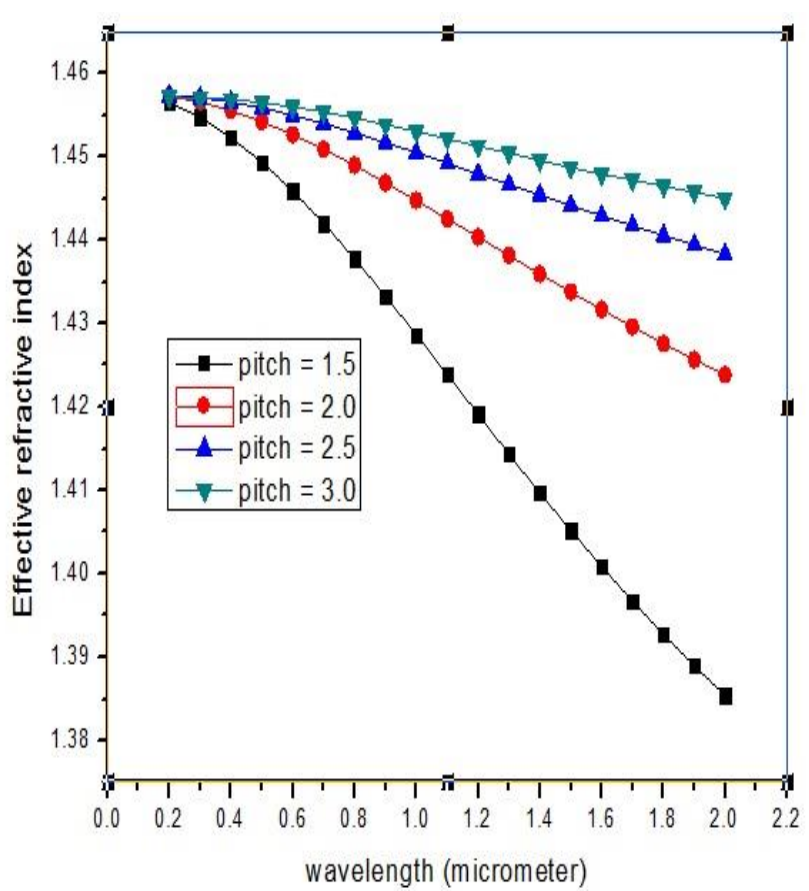

Figure: 7. Refractive index for constant air hole diameter having varying pitch.

Again the calculation is carried out for constant air hole diameter $(\mathrm{d}=1.0 \mu \mathrm{m})$ and for constant Pitch $(2.0 \mu \mathrm{m})$. but this time rings are varying.

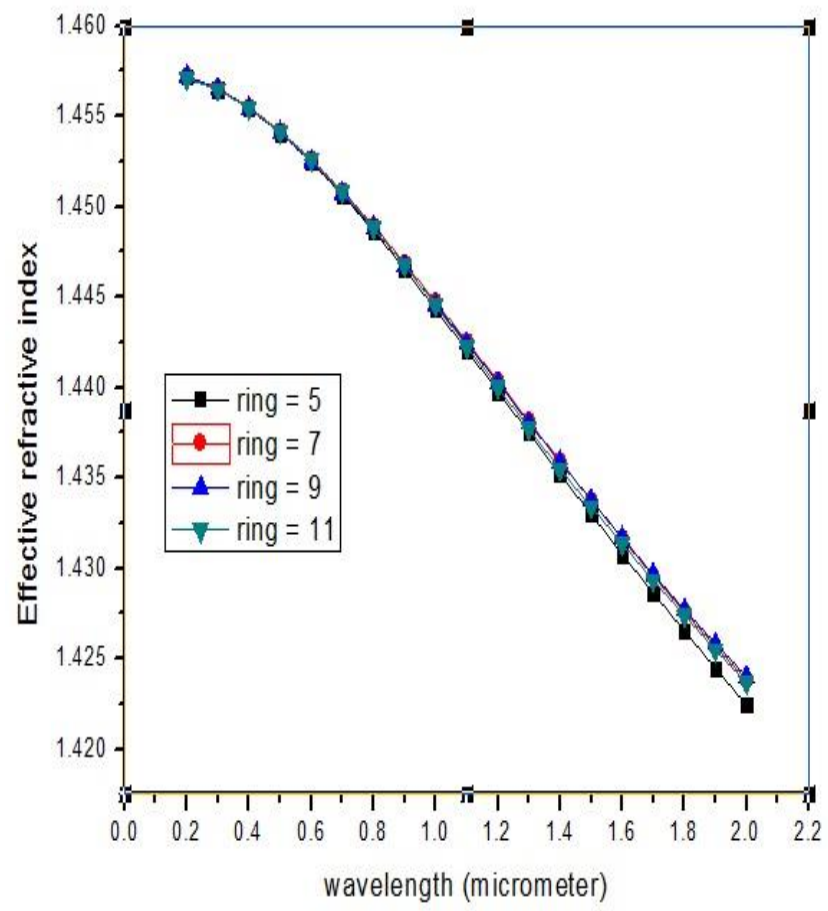

Figure: 8. Refractive index for Constant air hole diameter and constant Pitch.

The calculation of dispersion for honeycomb PCF for various hole diameter and pitch size is calculated and the result is shown below.

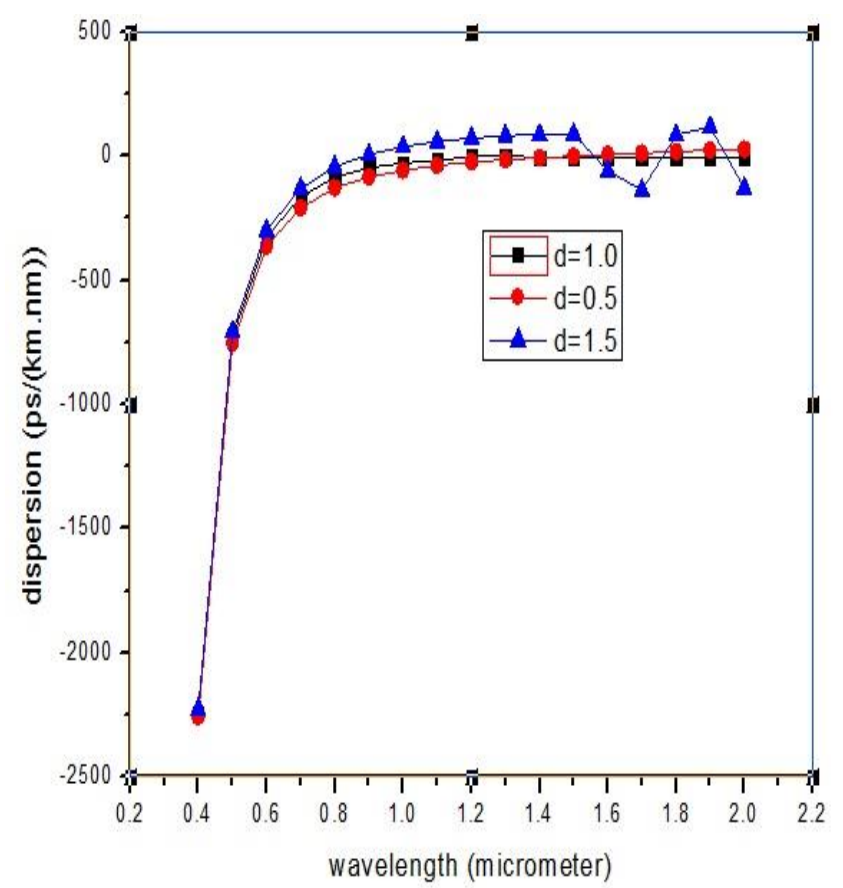

Figure: 9. shows the comparison of chromatic dispersion of proposed Honeycomb PCF when pitch is $2.0 \mu \mathrm{m}$.

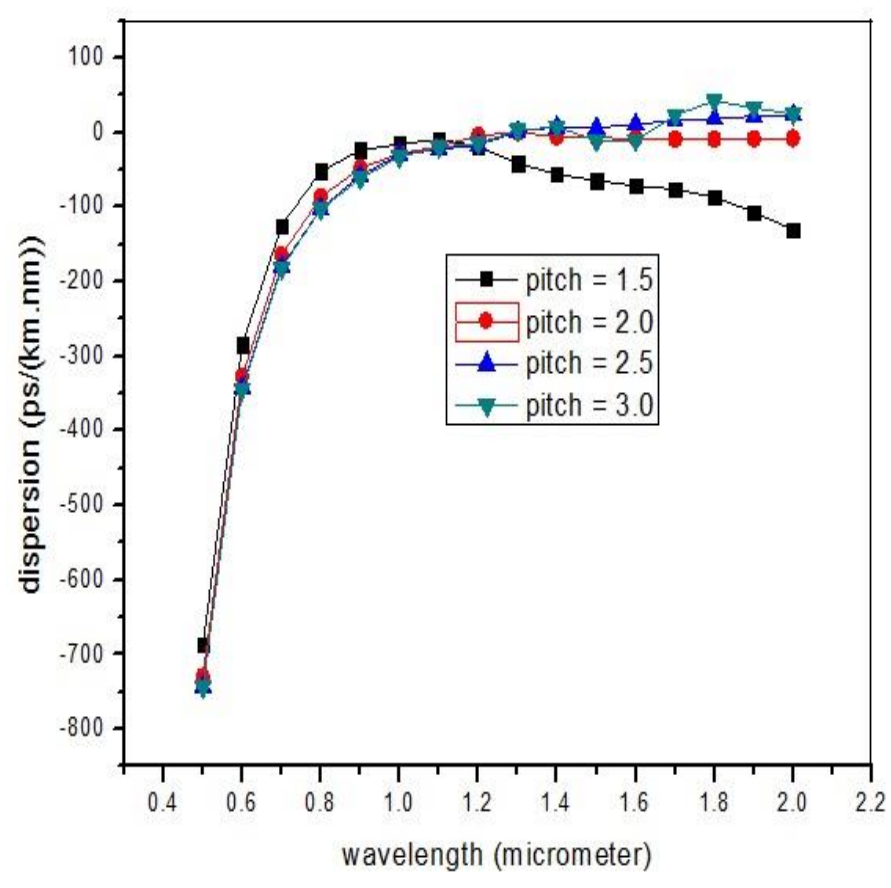

Figure: 10. Comparison of chromatic dispersion of proposed Honeycomb PCF when air hole diameter is $1.0 \mu \mathrm{m}$. 


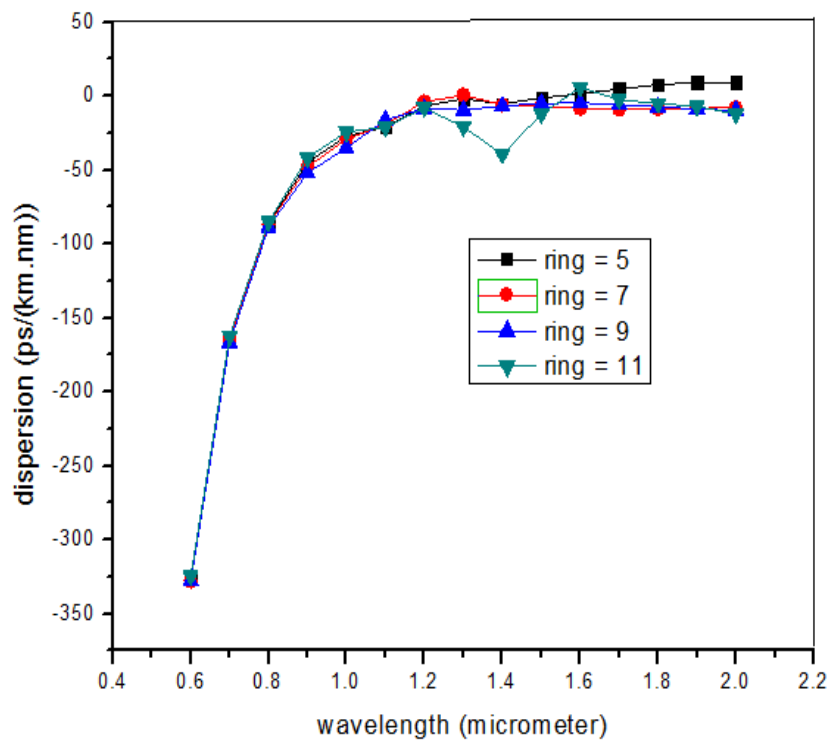

Figure: 11. Shows the comparison of chromatic dispersion of proposed Honeycomb PCF when pitch is $2.0 \mu \mathrm{m}$ and air hole diameter $(\mathrm{d})$ is $1.0 \mu \mathrm{m}$.

From the above discussion we have found that best dispersion result can be calculated for air hole diameter $(\mathrm{d})=1.0 \mu \mathrm{m}$ Pitch $=2.0 \mu \mathrm{m}$.

\section{CONCLUSION}

The result, so obtained, gives that the dispersion calculated for Honeycomb photonic crystal fiber using the Scalar index method gives best result in comparison of other structures. Here we have calculated the dispersion for various data but it shows that when we consider air hole diameter $1.0 \mu \mathrm{m}$ and Pitch $2.0 \mu \mathrm{m}$ and select the 7 layers then it gives best result. The fiber parameters are optimized to yield best agreement with available data. The discrepancy observed at higher wavelength values is due to the fact that the refractive index of the silica and the effective cladding index are wavelength dependent.

\section{ABBREVIATIONS AND ACRONYMS}

HPCF: Honeycomb Photonic Crystal Fiber

TBC: Transparent Boundary Condition.

SEIM: Scalar Effective index method.

UFD: Ultra Flattened Dispersion.

RI: Refractive Index.

EIM: Effective Index method.

FSM: Fundamental space-filling mode.

\section{ACKNOWLEDGEMENT}

The author acknowledges to his co-authors for their kind support and help during the research on such type of material. Basically this work is done on SEIM and our co-author done a lot of research on this topic. The author also show his gratitude to Prof. R. K. Aeron, Director, Pacific Institute of Technology, and Prof. S. K. Sharma, Director Pacific Institute of Engineering for their support throughout during this work.

\section{REFERENCES}

[1].Knight, T.A. Birks, P. St. J. Russel, and D.M. Atkin, “ All silica singlemode optical fiber with photonic crystal cladding", Opt. Lett, 21, pp. 1547 - 1549 (1996).

[2] J. Broeng, D. Mogilevstev , S.E. Barkou, and A. Bjarklev, "Photonic crystal fibers: a new class of optical waveguides", Optical fiber Technology, 5, pp. 305-330(1999).

[3] J.C. Knight, T.A. Birks, P.St.J. Russell and J.P. Sandro, "Properties of photonic crystal fiber and the effective index mode", J.Opt,Soc. Am, 15,1998, pp748-752.

[4]R.K.Sinha, S.K.Varshney, "Dispersion properties of photonic crystal fibers",Microwave Opt. Technol. Lett.vol 37,2003,pp 129-132.

[5]Shailendra K. Varshney,M.P.Singh,R.K.Sinha, "Propagation characteristics of photonic crystal fibers",Opt. Comm.vol 24,2003,pp 856.

[6].Chen, D., M.-L. Vincent Tse, and H. Y. Tam, ISuper-lattice structure photonic crystal ${ }^{-}$ber," Progress In Electromagnetics Research M, Vol. $11,53\{64,2010$.

[7]. Wiederhecher, G. S., C. M. B. Cordeiro, F. Couny, F. Benabid, S. A. Maier, J. C. Knight, C. H. B. Cruz, and H. L. Fragnito, IField enhancement within an optical - bre with a subwavelength air core," Nature Photonics, Vol. 1, 115\{118, 2007.

[8]. Dudley, J. M. and J. R. Taylor, ITen years of nonlinear optics in photonic crystal - bre," Nature Photonics, Vol. 3, 85\{90, 2009.

[9]. F. Gerome, "Design of dispersion-compensating fibers based on a dual-concentric-core photonic crystal fibers", Opt .Lett. 29, pp. 2725-2727, Dec. 2004.

[10]. F.Poli, "Tailoring of Flattened Dispersion in High Nonlinear Photonic Crystal Fibers", IEEE Photon. Tech. Lett. vol. 16. , no 4, pp. 1065-1067, Apr. 2004.

[11]. S. S. Mishra and Vinod Kumar Singh, Study of Nonlinear Property of Hollow core Photonic crystal fiber," International Journal of Light and Electron Optics 122(8) pp.687-690, 2011.

[12]. L. Vincetti, "Confinement Losses in Honeycomb Fiber", IEEE Photon. Tech. Lett. 16, pp. 2048-2050, 2004.

[13]. L. Vincetti, F. Poli, and S. Selleri, "Confinement loss and nonlinearity analysis of air-guiding modified honeycomb photonic bandgap fibers", IEEE Photon. Technol. Lett. 18, pp.508-510, 2006.

[14]. M. Chen and R. Yu, "Analysis of photonic bandgaps in modified honeycomb structures," IEEE Photon. Technol. Lett. 16, 819-821 2004

[15] Gangi R. Salla, Physical Research Laboratory, India Photonics 2012.

[16] Yan-feng Li *, Ching-yue Wang, Ming-lie Hu. Tianjin 300072, China

[17] Ravindra Kumar Sharma, Kirti Vyas, Ajay Kumar Bairwa "Flattened Dispersion of Hexagonal Chalcogenide As2Se3 Glass Photonic Crystal Fiber with a Large Core.”, May-2012, pp 201-203.

[18] Ravindra Kumar Sharma, Kirti Vyas and Navneet jaroli

"Investigation of Zero Chromatic Dispersion in Square Lattice As2Se3Chalcogenide Glass PCF”,pp 23 - 26, July-2012.

[19] Anshu D. Varshney*a, S.K.Varhsney** and R.K.Sinha* Modal Analysis of Photonic Crystal Fiber by Scalar and Fully Vectorial Effective Index Methods.

Ravindra kumar Sharma: He has done his B.E. in Electronics \& Communication from university of Rajasthan and completed his M.Tech. from Rajasthan Technical university in Digital Communication.

Sunil Sharma: He has done B.E. HONS. From University of Rajasthan, Jaipur in Electronics \& Communication. Recently he is associated with Pacific University.

Shambhu Dayal Sharma, Pursuing in M.Tech., MNIT, Jaipur. 